\title{
COMPARISON OF QINZHOU BAY WETLAND LANDSCAPE INFORMATION EXTRACTION BY THREE METHODS ${ }^{1}$
}

\author{
CHANG Xiao-li ${ }^{\mathrm{a}}$ ZHANG Qiang ${ }^{\mathrm{b}} \quad$ LUO Ming-liang ${ }^{\mathrm{a} *}$ DONG Cheng-y an ${ }^{\mathrm{a}}$ \\ ${ }^{a}$ Land and Resources College, China West Normal University, Sichuan Nanchong-991344756@qq.com. \\ ${ }^{\mathrm{b}}$ Qingzhou University, Guangxi Qinzhou -294836522@qq.com
}

KEY WORDS : coastal wetland extraction; Supervised Classification; Decision Trees; Bject-oriented; Qingzhou Bay

\begin{abstract}
ABS TRACT:
Wetland ecosystem plays an important role on the environment and sustainable socio-economic development. Based on the TM images in 2010 with a pretreament of Tasseled Cap transformation, three different methods are used to extract the Qinzhou Bay coastal wetlands using Supervised Classification (SC), Decision Trees (DT) and Object-oriented (OO) methods. Firstly coastal wetlands are picked out by artificial visual interpretation as discriminant standard. The result shows that when the same evaluation template used, the accuracy and Kappa coefficient of SC, DT and OO are 92.00\%, 0.8952;89.00\%、0.8582; $91.00 \% 、 0.8848$ respectively. The total area of coastal wetland is $218.3 \mathrm{~km}^{2}$ by artificial visual interpretation, and the extracted wetland area of SC, DT and OO is $219 \mathrm{~km}^{2}, 193.70 \mathrm{~km}^{2} 、 217.40 \mathrm{~km}^{2}$ respectively. The result indicates that SC is in the first place, followed by OO approach, and the third DT method when used to extract Qingzhou Bay coastal wetland.
\end{abstract}

\section{INTRODUCTION}

Wetlands is a unique ecosystem which is formed by the interaction between water and land, being one of the important natural habitats and ecological landscapes whose biodiversity is the richest (Yang, 2002a). According to preliminary statistics, the total wetlands area of China is about 6,594 million $\mathrm{hm}^{2}$, about $10 \%$ of the world's wetland area (Wu et al, 2007). Using RS technology to extract the information of wetland timely and accurately is an important base for Wetland Research. There are currently a large number of research results, almost covering the most aspects of wetland research, mostly focusing on wetland definition and classification(Yang,2002), wetland formation and characteristics, resource distribution, wetland eco-tourism, landscape, tourism development and wetlands protection, etc(Yang, 2002b). In recent years, the researchers are always focusing on the following topics such as the physical, chemical, biological processes and mechanisms of wetland ecosystems; relationship between the processes and the functions (Yang, 2002b); the concept of wetland ecosystem's health, diagnosis indexs, meantime devoting to improvement the ability of early warning(Sun et al , 2013).

Due to the unique and complex nature of the wetland ecosystem, visual interpretation is used to be the main method of wetland information extraction with high precision. But this method often takes more manpower and plentiful time (Yang,2002b). The implementation of interactive wetland information extraction is a hot spot in current research based on RS software platform of Erdas. Many interactive methods have been used such as supervised classification (SC), decision tree (DT) and object-oriented (OO) method to extract wetland information (Huan et al, 2009; Shen et al, 2007). Due to different understanding and different purpose of information extraction, as well as the diversity of wetlands, a extensive distribution and variability of water environment, etc., one single method used only to extract wetland information is often difficult to

* Corresponding author: LUO Ming-liang. E-mail:Lolean586@163.com。 
obtain the desired results (Yin et al, 2010). This article selects qinzhou bay as the sample area, comparing and analyzing the difference of SC, DT and OO, which are often used to extract wetlands information.

\subsection{The study area}

Qinzhou Bay locates in the south of Qinzhou City, the south of the Guangxi Zhuang Autonomous Region, belonging to the $\mathrm{BeiBu}$ Gulf, and it is one of the most important part of the Beibu Gulf. The connection between Damiandun (Tortoiseshell Island) at south of Rhino Feet Peninsula and Tiantang Corner of Qisha Peninsula is the south border of Qinzhou Bay. The north of Qinzhou Bay is MaoWeiHai with greatly developed aquaculture. There are hundreds hectares of Mangrove Forests growing in the region. Diurnal tides is he main type in Qinzhou
Bay with an average annual temperature $21.3^{\circ} \mathrm{C}$. According to the status quo of China's wetland classification system, "Convention on Wetlands" and "national wetland resources investigation and monitoring of technical regulations", Qinzhou Bay wetlands in southern China is a typical coastal wetlands.

There are various types of coastal wetland in qinzhou bay, including tidal flats wetland, river wetland, mangrove (Composed of mangrove, disadvantage, autumn in tomato, olive wood, red sea lam, lacquer tree species composition and so on) and all other kinds of artificial breeding farm in tidal flats or not in tidal flats. According to the principles of science, regionalism, readability and consistency, combination with the actual situation in Qinzhou Bay Wetlands (Li et al, 2012), Qinzhou Bay Wetland can be classified as table 1 .

\begin{tabular}{|c|l|}
\hline Wetland types of Level 1 & \multicolumn{1}{|c|}{ Wetlands Types Of Level 2 } \\
\hline Natural wetlands & $\begin{array}{l}\text { Shallow sea waters, Tidal flats, Mangrove, River wetland, Natural vegetation } \\
\text { Aquaculture ponds, Coastal farms (such as Shrimp ponds), Reservoir, Paddy } \\
\text { field, Artificial vegetation }\end{array}$ \\
Artificial wetland
\end{tabular}

Table1. Classification systems of Qinzhou Bay Wetland

Since this study focuses on the coastal wetland, the key of information needed to extracted is as followed: tidal flats, farms, vegetation, rivers and seas. Among those elements, the tidal flats include beach wetland, mangroves and Shrimp ponds farms, while vegetation includes shelter forest, trees and natural forest. Artificial wetland is mainly refers to farms, fish farms formed by the pond wetland, such as shrimp ponds (although artificial trace existed, it still maintains the characteristics of wetlands). Because the horizontal resolution of TM images is only $30 \mathrm{~m}$, the sea, river and lake are unified into water bodies, while Tidal flats, mangroves, Shrimp ponds farms into coastal wetlands. Urban landuse types include urban land, village land, township construction land, industrial parks and roads. Agricultural landuse includes upland and paddy field. Finally the extracted information in this study can be divided into water, urban land, agricultural land, coastal wetland and vegetation.

\subsection{Data Sources And Pretreatment}

Landsat TM image data of Qinzhou in 2010 was used with the horizontal resolution of $30 \mathrm{~m}$. In order to get the boundaries of Qinzhou Bay, digital elevation models was used to clip the study area by watershed division.

$\mathrm{TC}$ transformation is also called the KT transformation, and its essence is a special method of principal component analysis. TC not only independs on a single image but also can compare soil brightness and green degree produced by different images, with the aim of image enhancement in extraction of vegetation information ( $\mathrm{Zi}$ et al, 2011). TC transformation was used to enhance the brightness, green degree and humidity of the image information.

\section{THE PROCESS AND METHODS OF WETLAND INFORMATION EXTRACTION}

\subsection{Technical Process}

Artificial visual interpretation, SC, DT and OO methods are used to extract Qinzhou bay wetland information, where artificial visual interpretation used to be the standard for accuracy evaluation. The whole technical process includes hierarchical classification, combining texture analy sis and other related indices, as figure 2 showed. 


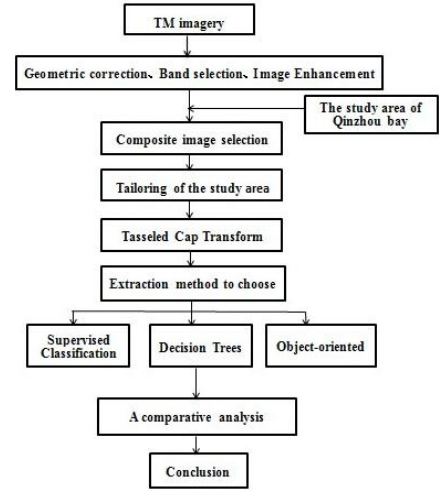

Figure. 2 Analy sis process

\subsection{S upervised Classification}

SC is often applied to more understanding of the situation in study area. Maximum likelihood classification also known as bayesian classification, is supervised classification based on image statistics, which is a typical and the most widely used in supervised classification method, and is also regarded as the highest accuracy classification in classification method (Dang et al,2010).

According to the characteristic of regional wetland, band combination of $(4,3,2)$ is chosen, meanwhile adaptive filter is used to reduce noise to highlight the boundary of wetland types without affecting information expression (Dang et al, 2010). The classification template is evaluated (figure 4.a, 4.b) based on classification template editor in Erdas Imagine and adjusting the classification property field before achieving the classification template information (figure 3). Supervised classification for pixels is performed based on category templates and classification with decision rules of the system default options (the Maximum Likelihood classification). One or more isolated pixels will appear in the classification results, which are need to clump, sieve, eliminate, recode etc(Dang et al,2010).

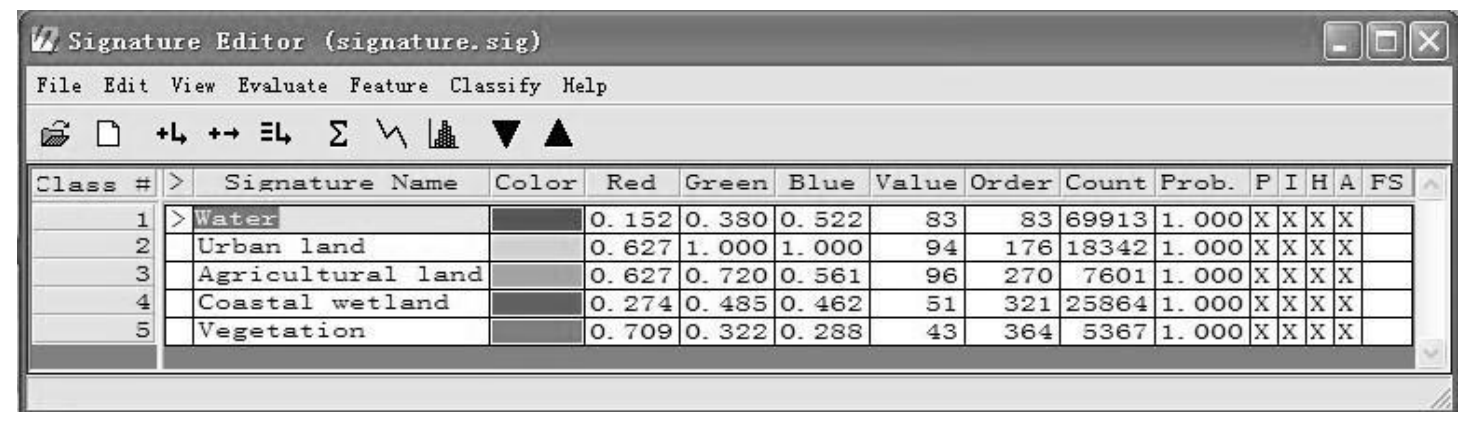

Figure. 3 Classification Template

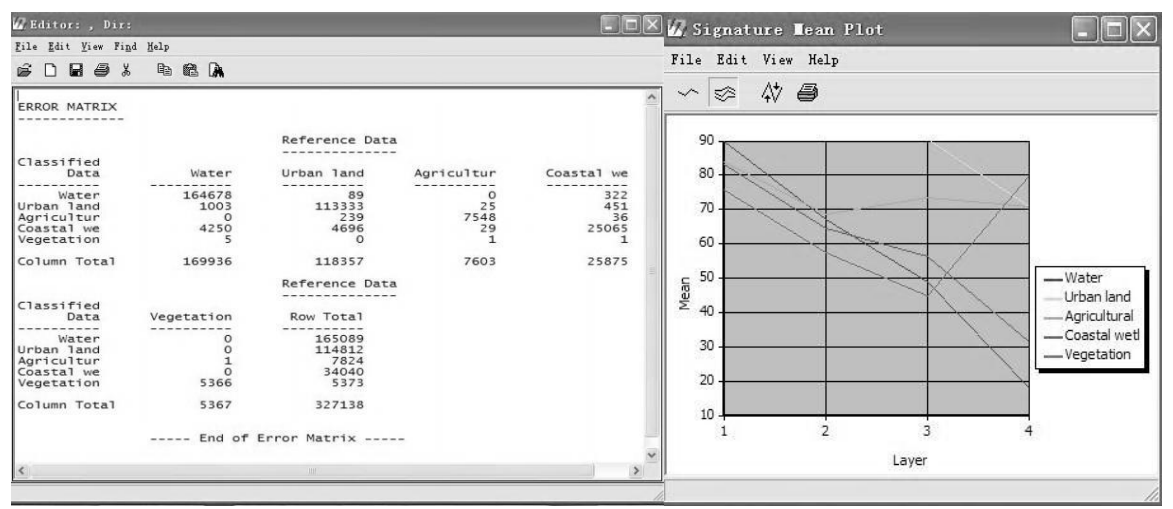

(a)

(b)

Figure. 4 classification template evaluation 


\subsection{Decision Tree Method}

Classification rules used to classify RS image can be produced by expert experiences, mathematical statistics and inductive methods in the process of DT classification based on knowledge (Li and Jia et al,2008).

Basic concept and elements of experts classifier are composed by decision tree, assumptions, rules, variable and related knowledge base. When image of band 4 selected, water is the most clear while tidal flats is the second one. When image of band 3 chosed, the vegetation and agricultural land are very clear while the others are fuzzy. According image clarity of the band and the pixel value in study area, decision rules are arranged as table 5 .

\begin{tabular}{|l|l|}
\hline water & TM Band $4>=5$ 且 $<=38$ \\
vegetation & TM Band $3>=10$ 且 $<=60$ \\
Agricultural land & TM Band $3>=55$ 且 $<=85$ \\
Coastal wetland & TM Band $1>=90$ 且 $<=255$ \\
Vegetation & TM Band $4>=35$ 且 $<=60$ \\
\hline
\end{tabular}

Table 5. Decision rules

The classification results can be viewed when region of interest chosen in knowledge classification and output option determined after knowledge base built by starting knowledge engineers, placing assumed elements to define rules, picking out the variables and testing knowledge base. Automatic classification will produce a lot of isolated pixel and it is necessary to eliminate and clump ( Dang et al,2010).

\subsection{Object-Oriented Method}

Object-oriented essentially differes to the traditional classification method based on pixels, for OO not dealing with single pixel, instead the whole image is regarded as a combination of different objects which can be interpreted ( Wang et al, 2009). OO utilizes spectrum information, texture feature and topology relations together to segment multiscale objects joint the thematic information. Fuzzy discrimination function is established through visual interpretation to classify different objects (Sun,Tong and Qin, 2008). OO is implemented based on software platform of ENVI and the process can be divided into two steps: finding object firstly and extracting feature followed. The steps for finding objects as following: image segmentation, block merge, block refining, computing object properties. Feature extraction can be achieved by supervised classification or classification based on rules. After many experiments (Yu and Zhan,2012;Hou et al,2010; $\mathrm{Li}$ etal,2008;Huang et al,2010;Xun etal,2013), the segmentation and merging threshold used are 51 and 48.3 respectively. The process of $\mathrm{OO}$ can be organized as figure 6 .

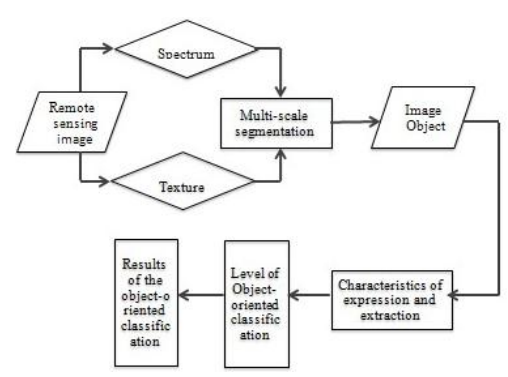

Figure. 6 Flow chart of $\mathrm{OO}$

\section{RES ULT and EVALUATION}

In RS image classification, the evaluation indices often used are as followed, the overall classification accuracy, Kappa coefficient, Commission Errors, Omission Errors. Confusion Matrix (CM) is used to compare classification results and the real information of terrain reflected by RS image. In this study, the accuracy of analysis is all utilized by ERDAS. Firstly Stratified Random distribution is used to pick out 200 pixles randomly from RS image, and comparison of information for every point is preformed between extracted information and real one by artificial visual discrimination. Classification confusion matrix, classification accuracy and Kappa coefficient of those 200 pixels are exported by the Accuracy Assessment Analy sis Tool.

\begin{tabular}{|c|ccccc|}
\hline Class Name & reference Totals & Classified Totals & Number Correct & Producers Accuracy & Users Accuracy \\
\hline Undefined & 1 & 1 & 1 & -- & -- \\
Water & 70 & 70 & 66 & $94.29 \%$ & $94.29 \%$ \\
Urban land & 27 & 29 & 22 & $81.48 \%$ & $75.86 \%$ \\
Agricultural land & 45 & 45 & 45 & $100.00 \%$ & $100.00 \%$ \\
Coastal wetland & 16 & 19 & 36 & $87.50 \%$ & $73.68 \%$ \\
Vegetation & 40 & 36 & 184 & $90.00 \%$ & $100.00 \%$ \\
Totals & 199 & 200 & & & \\
\hline
\end{tabular}


Table 7 . Accuracy Evaluation of TC

\begin{tabular}{|c|ccccc|}
\hline Class Name & Reference Totals & Classified Totals & Number Correct & Producers Accuracy & Users Accuracy \\
\hline Undefined & 68 & 67 & 67 & --- & -- \\
Water & 24 & 30 & 24 & $100.00 \%$ & $80.00 \%$ \\
Urban land & 14 & 23 & 11 & $78.57 \%$ & $47.83 \%$ \\
Agricultural and & 8 & 4 & 4 & $50.00 \%$ & $100.00 \%$ \\
Coastal wetland & 36 & 33 & 30 & $83.33 \%$ & $90.91 \%$ \\
Vegetation & 50 & 43 & 42 & $84.00 \%$ & $97.67 \%$ \\
Totals & 200 & 200 & 178 & & \\
\hline
\end{tabular}

Annotation: Overall Classification Accuracy $=89.00 \% \quad$ Overall Kappa Statistics $=0.8582$

Table 8. Accuracy Evaluation of DT

\begin{tabular}{|c|ccccc|}
\hline Class Name & Reference Totals & Classified Totals & Number Correct & Producers Accuracy & Users Accuracy \\
\hline Undefined & 64 & 64 & 64 & --- & -- \\
Water & 36 & 40 & 34 & $94.44 \%$ & $85.00 \%$ \\
Urban land & 11 & 14 & 6 & $54.55 \%$ & $42.86 \%$ \\
Agricultural land & 12 & 6 & 6 & $50.00 \%$ & $100.00 \%$ \\
Coastal wetland & 32 & 33 & 30 & $93.75 \%$ & $90.91 \%$ \\
Vegetation & 45 & 43 & 42 & $93.33 \%$ & $97.67 \%$ \\
Totals & 200 & 200 & 182 & --- & -- \\
\hline
\end{tabular}

Annotation: Overall Classification Accuracy $=91.00 \% \quad$ Overall Kappa Statistics $=0.8848$

Table9 . Accuracy Evaluation of OO

When the same evaluation template is used, total accuracy of SC, DT and OO are $92.00 \%, 89.00 \%$ and $91.00 \%$ respectively, while Kappa coefficient is $0.8952,0.8582$ and 0.8952 respectively.

The totoal accuracy of SC can reach as high as $92.00 \%$. The main reasons maybe relate with the following factors, including familiar with the study area, the maximum likelihood method used. The bands used in study are the combination of band (4 3 2) of TM images, which results in that waters are dark blue while vegetation appears red. So it is easy to select training area to achieve high accuracy. While since it is easily confused among agricultural land, urban land, water and coastal wetland with $30 \mathrm{~m}$ resolution TM images, meanwhile a mixture of various land types exists in the training area, the classification accuracy is reduced to some extent.

The establishment of the knowledge base is the precondition of the DT methods, the definition of rules and the determination of variable are the key in wetland extraction. Water and coastal wetland choose the same band of 4 , and wavelength ranges in 5-38 and 35-60 respectively. Since the spectral characteristics of artificial breeding farms, such as shrimp pounds, seems like water surface, which leads to a less obvious results, as it shows that water is confused with artificial breeding farm.

OO based on ENVI can effectively identify water, construction and other features, which is because that not only the object shape and structure characteristic used, but also shape and texture of the image taken into account together. It is in this sense that the conflicts such as "same body different spectrum" and "foreign body with spectrum" are resolved in a certain extent. The key factor of $\mathrm{OO}$ is the threshold selection in the process of image segmentation and merging. Since it is difficult to obtain the optimal image segmentation scale (Dang et al, 2010) that a small amount of water confused with coastal wetland, which affects the precision of classification. In addition, OO method is good at recognizing high resolution image while TM data used only with a horizontal resolution of $30 \mathrm{~m}$, that affectes the accuracy of coastal wetland information. But on the whole, it still reachs the classification accuracy of $91 \%$.

The comparisons of three methods used are as shown in table 


\begin{tabular}{|c|c|c|c|}
\hline & $\mathrm{SC}$ & DT & $\mathrm{OO}$ \\
\hline advantages & $\begin{array}{l}\text { Highlight the characteristics of wetland, } \\
\text { resulting in good classification results in } \\
\text { subclass of Wetlands. } \\
\text { The operation is simple, can be } \\
\text { implemented in the ENVI and ERDAS }\end{array}$ & $\begin{array}{l}\text { Define the classification rules; The } \\
\text { small amount of calculation } \\
\text { relatively; It can display the } \\
\text { importance of the field; }\end{array}$ & $\begin{array}{l}\text { the extraction results } \\
\text { objectively, figure } \\
\text { boundary smoothing, object } \\
\text { feature rich }\end{array}$ \\
\hline disadvantages & $\begin{array}{l}\text { the Spectral characteristics of demand is } \\
\text { high }\end{array}$ & Continuity field hard to predict & $\begin{array}{l}\text { difficult to obtain the } \\
\text { optimal segmentation scale }\end{array}$ \\
\hline Suitable range & $\begin{array}{l}\text { The area Affected by the phonological } \\
\text { characteristics is not big }\end{array}$ & $\begin{array}{l}\text { Various types of wetlands and } \\
\text { area is large }\end{array}$ & $\begin{array}{l}\text { Various types of wetlands } \\
\text { and area is large }\end{array}$ \\
\hline advice & $\begin{array}{l}\text { Combining with field spectral } \\
\text { characteristics }\end{array}$ & brief attribute before classification & $\begin{array}{l}\text { Select appropriate } \\
\text { segmentation scale }\end{array}$ \\
\hline
\end{tabular}

Table10. Comparison of SC, DC and OO

The total area of study area is $2542.00 \mathrm{~km}^{2}$, among which the total area of coastal wetland through a visual discrimination is $218.30 \mathrm{~km}^{2}$, accounting for about $8.59 \%$ of total area. Wetland areas of three methods are $219 \mathrm{~km}^{2}, 193.7 \mathrm{~km}^{2}, 217.4 \mathrm{~km}^{2}$ respectively. The result shows that extraction of wetland area through SC is most similar with visual interpretation with a more area of $0.7 \mathrm{~km}^{2}$, while $\mathrm{OO}$ resulting in $0.9 \mathrm{~km} 2$ missing and DT $28.3 \mathrm{~km}^{2}$ missing. On the whole both classification accuracy and Kappa coefficient of supervised classification are higher than the other two methods.

In general, OO classification method should be optimal. While in this study, the coarse horizontal resolution of TM image and the particularity of coastal wetlands will be the main factors influencing the classification accuracy. As it known that horizontal resolution of TM image is only $30 \mathrm{~m}$, while existed research shows that $\mathrm{OO}$ approach will get the best results when $10 \mathrm{~m}, 0.61 \mathrm{~m}$ or $0.5 \mathrm{~m}$ horizontal resolution image used with the classification accuracy as high as 90\% (Yu and Zhan,2012;Hou et al,2010;Li etal,2008;Huang et al,2010;Xu etal,2013).

Secondly, QinZhou bay wetlands are mainly distributed in MAO tail sea - LongMen islands - inland sea area of longMen port and the coast of south sea area of longMen port. The governed types of wetland are as followed, such as beaches, tidal flats hy grophilous mangroves, fish (or shrimp) ponds, the big oyster beds of Oyster row. The amount of wetland types and area Inland sea are bigger than offshore area. From the viewpoint of spatial distribution, the main aggregated wetland includes farms, tidal flats and mangrove forests. Oyster row concentrated at Maowei sea - the mouth of longMen Port and the Shrimp (or fish) ponds partly distribute at the northeast shore of MAO wei sea and the big ring village. The main type which distributes discretely is shrimp ponds near the shoreline. The size of every oyster rafts ranges between $20 \mathrm{~m}$ and $40 \mathrm{~m}$, while oyster rafts connected into a large scale pieces. The average size of shrimp pounds is at about $25 * 25 \mathrm{~m}$. Obviously TM 30m horizontal resolution is insufficient to express shrimp discrete distribution, thus resulting in lower classification accuracy of $\mathrm{OO}$.

From the viewpoint of spatial location, the water is assigned into the wetland through SC as a mistake, consequently resulting in the increasing of wetland area. The typical case is that an isolated Gold Nest Reservoir and a small part of Dafeng River Basin divided into wetlands. Some scattered wetlands (average size about $2.44 * 10^{-4} \mathrm{~km}^{2}$ ) produced by OO method, are divided into water bodies, mainly distributed in MAO sea, while in the results of DT the naccuracy region scattering along rivers and estuary partly with the average area about $2.70 * 10^{-4}$ $\mathrm{km}^{2}$.

Some interpretation error happens in the sub-type of wetland, such as Gold Nest Reservoir is interpreted into the wetland, while the oyster rows into urban landuse by SC. The oyster rows, tidal flats and mangrove forests of longmen islands are divided into water by DT. MAO tail farms (mainly to shrimp pounds) are interpolated into farmland or water, which locates in the north bank of Maowei Sea by OO. And other inaccurate classification by $\mathrm{OO}$ is that tidal flats mangrove is categorized into water at the shore of Maowei Sea. The finnal classification by SC, DT and OO is shown in Figure 11.b, 11.c and 11.d, while Figure 11.a is the original TM images.

Although the total classification accuracy of SC is higher than 
other methods, while accuracy of the producers and the users of SC are the lowest. On the contrary, the OO methods has the highest accuracy as table 3-5 shown. The texture feature of shrimp ponds and oyster rows is obvious, so those feature can be picked out more easily by OO. Although it is difficult for DT to define the threshold of the wetland and water, since in the value of 35 to 38 water and wetlands are mixed together, which mainly reflected in the oyster rows and Shrimp ponds. But in general the water and wetlands can be separated apart. $\mathrm{SC}$ is mainly according to sample pixels. Since the differences of tidal flats, mangroves and Shrimp ponds are bigger than other, then they are easier to be identified, while oyster rows is similar with water, their accuracy is not good as the features above.

\section{CONCLUSION AND DISCUSSION}

A comparison of SC, DT and OO used to extract QinZhou Bay wetland after TC transformation has been done, and the result shows that a certain accuracy of wetland information can be obtained by any one in the three methods. The suitable method should be selected according to specific data accuracy and classification requirements.

When the same classification characteristics and the premise of accuracy evaluation samples used, the accuracy and Kappa coefficient of the three methods are $92.00 \%$ and 0.8952 , $89.00 \%$ and $0.8582,91.00 \%$ and 0.8848 respectively. The results indicate that SC used to extract Qinzhou Bay wetland will achieve the best overall effect.

In the process of coastal wetland information extraction, a small number of wetland and water mixed together, and the $\mathrm{OO}$ method does not show its advantage when $30 \mathrm{~m}$ resolution TM images used. Meanwhile in Wetland classification process, the setting of parameters and thresholds mainly depend on the person's subjective experience. How to accurately determine the Segmentation and merging of the scale of the threshold still need a large number of trials. In addition, how to use the image texture information effectively also need to be further excavated.

\section{ACKNOWLEDGEMENT}

The author would like to thank the financial support from national natural science foundation of China under grant 41101348, application foundation development program of science and technology departure in Sichuan Province under grant 2010JY0089, key project in natural science of education department in Sichuan Province under grant 2009ZA120.

\section{References:}

Du hongy an Zhang Hongy an, Zhang Zhengxiang, 2004. Under the GIS support of wetland remote sensing information and high precision classification method research. Remote sensing technology and applications, 19 (4), pp.244-248.

Dang anrong, Jia Haifeng Chen Xiaofeng etc, 2010. ERDAS IMAGINE - remote sensing image processing tutorial. Beijing: tsinghua university press.

Huang Ying Zhou Yunxuan Wuwei, etc, 2009. Shanghai urban wetland remote sensing extraction and classification based on decision tree model. Journal of Jilin University (Earth Science Edition), 33 (6), pp. 1156-1162.

Houwei, LuXueJun Zhang Chunxiao etc,2010. Study on the method of object-oriented high-resolution image information extraction --use extraction of Sichuan wenzhou residents as example. J Geo-Information Science, 12 (1), pp.119-25

Huangliang ZuoXiaoQing Zhang Xiaoxiao etc, 2010. Object-oriented of road information analysis of identification and extraction. Journal of Kunming University of Science and Technology (Science and Technology), 35 (6):6-10.

Li Fangfang Jia YongHong, 2008. A wetland information extraction method and its change detection based on TM images. Science of Surveying and Mapping (2) , pp.147-149.

Li min, Cui Shiyong, Li chengming, etc, 2008. The object-oriented high-resolution remote sensing image information extraction-- use extracted cultivated land as example.Remote sensing information, (6) , pp. 63-6+89

Li Dongying Yang Wenbo, wanglin etc ,2012. Base the RS data of the Yellow River delta wetland information extraction and wetland dynamics study. Transaction of Oceanology and Limnology, (1), pp.100-109.

ShenWenMing wang wenjie Luo Haijiang, etc, 2007. The remote sensing image classification method based on decision tree classification technology research. Remote sensing technology and applications, 22 (3) , pp.333-338.

Sun Junjie Ma Daxi, Ren Chunying etc, 2013. Environmental satellite data based on long phase of south urn river wetland information extraction method research .Wetland Science, 11 (1), pp.60-67.

Sun Yongjun TongQingXi, Qin Jiming, 2008. Using the object-oriented method to extract wetland information. Remote Sensing for Land \& Resources, pp.79-82+ 108 
wu hui Deng Yulin Li Chunyan etc,2007. The wetland research, protection and development in China. World forestry research, 20 (6), pp.42-49.

Wang FeiFei Wei Jianbing, Wenya,etc, 2009. The review of Wetland remote sensing information extraction methods . The national land and resources and environment remote sensing application technology conference, China shenzhen, guangdong province, $\mathrm{F}$.

Xu Jingping, Zhao Jianhua, Zhang Fengshou, etc, 2013.The object-oriented pond farming with the sea information extraction.Remote sensing for Land \& Resources, 25 (1), pp. 82-3-4-5.

Yang Yongxing,2002a. International wetland science research progress and the Chinese wetland science research priority areas and prospect. Advances in Earth Science,

(4),pp.508-514.
Yu Xiaomin, Zhan feibing, 2012. Based on high resolution remote sensing image of urban surface information extraction research, Geomatics \& Spatial Information, 35 (7) , pp.21-24.

Yang Yongxing, 2002b. The main characteristics, progress and future of international wetland scientific research, .Advances in Earth Science, (2), pp.111-120.

YanShuBai Lv Xianguo, Wu Haitao, 2010. Some theory problems in the study of wetland definition. Wetland science, 8 (2), pp.182-188.

Zihui, Qin Yong, Yanghui, etc, 2011. Coastal wetland information extraction method comparative study. Computer engineering and applications, 47 (33), pp. 244-248.

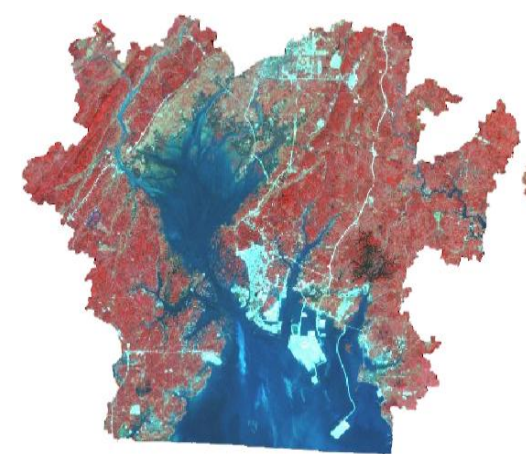

a

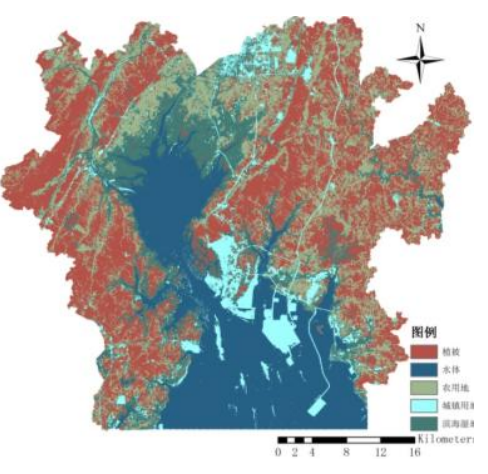

b

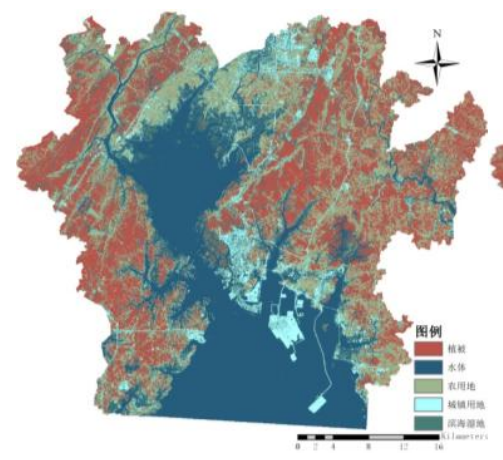

c

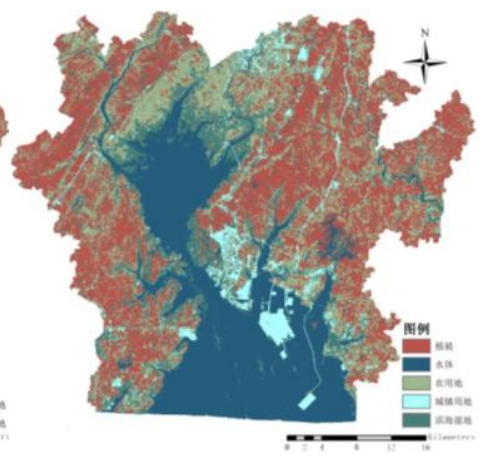

d

Figure 11. The extraction results contrast 